\title{
n-3 LC-PUFA Enrichment Protocol for Red Earthworm, Eisenia fetida: A Cheap and Sustainable Method
}

\author{
Metin Kumlu ${ }^{1,2}\left(\mathbb{D}^{-}\right.$, Asuman Beksari ${ }^{1,2}$, Hatice Asuman Yilmaz ${ }^{2}$, Merve Sariipek $^{3}(\mathbb{D}$ \\ Enes Kinay ${ }^{2}$, Giovanni M. Turchini ${ }^{4} \oplus^{\infty}$, Orhan Tufan Eroldogan ${ }^{2, *}$
}

\author{
${ }^{1}$ Mems Karides Ltd. Şti., Baharlı Street. No: 023, Milas, Muğla, Turkey. \\ ${ }^{2}$ Çukurova University, Faculty of Fisheries, 01330 Balcalı, Adana, Turkey. \\ ${ }^{3}$ Sinop University, Faculty of Fisheries, Sinop, Turkey. \\ ${ }^{4}$ Deakin University, School of Life and Environmental Sciences, Waurn Ponds Campus, Geelong, Victoria, Australia.
}

\section{How to cite}

Kumlu, M., Beksari, A., Yilmaz, H. A., Sariipek, M., Kinay, E., Turchini, G. M., Eroldogan, O. T., (2021). n-3 LC-PUFA Enrichment Protocol for Red Earthworm, Eisenia fetida: A Cheap and Sustainable Method. Turkish Journal of Fisheries and Aquatic Sciences, 21, 333-346. http://doi.org/10.4194/1303-2712-v21_7_03

\section{Article History}

Received 22 October 2020

Accepted 30 March 2021

First Online 07 April 2021

Corresponding Author

Tel.: +905337783004

E-mail: mtufan@cu.edu.tr

\section{Keywords}

Fish oil

DHA

22:6n-3

n-3 LC-PUFA

Eisenia fetida

Broodstock diet

\begin{abstract}
This study assessed the potential of omega- 3 long chain polyunsaturated fatty acids ( $n$-3 LC-PUFA) enrichment in red earthworm (Eisenia fetida, REW), via the addition of fish oil (FO, anchovy oil) to the culture-compost. FO addition to compost was tested at graded inclusion doses $(0,2.5,5$ and $10 \mathrm{~g} / 100 \mathrm{~g}$ compost) and for different timeperiods, up to $96 \mathrm{~h}$. Dose, time, as well as dose and time interactions of FO compost enrichment had significant effects on all fatty acids of earthworm bodies. The enrichment with $10 \mathrm{~g} \mathrm{FO} / 100 \mathrm{~g}$ compost sharply increased DHA levels of the worms (14.01 mol\%) within just $24 \mathrm{~h}$, compared to the control group (0.92 mol\%). Similar increases (ranging between 10.99 and 15.55 mol\%) occurred only after 48 and $96 \mathrm{~h}$ in lower FO enrichment levels ( 2.5 and $5.0 \mathrm{~g} / 100 \mathrm{~g}$ compost, respectively). Therefore, it was concluded that, to obtain maximum n-3 LC-PUFA enrichment efficiency in REW, an enrichment period of $24 \mathrm{~h}$ is ideal and recommended for the $10 \mathrm{~g} \mathrm{FO} / 100 \mathrm{~g}$ compost, whereas longer enrichment periods (48-96 h) are more suitable for lower levels of FO inclusions ( 2.5 or $5 \mathrm{~g} / 100 \mathrm{~g}$ compost).
\end{abstract}

\section{Introduction}

Fish meal (FM) and fish oil (FO) are two important raw materials used in aquaculture feed, as they efficiently fulfil the dietary needs of both essential amino acids and essential fatty acids for cultured fish and crustaceans (Medale \& Kaushik, 2009; Turchini et al., 2009; Turchini, 2013; Jobling, 2016). However, because of their limited supply and increasing demand, nutritionally balanced and low-cost alternative resources have been sought after and researched intensively by the aquaculture research and development sector. In this context, earthworms, which have been utilised as live bait in the hunting of fishes in the United States since 1940's (Mason et al., 1992) and used globally in the aquarium industry for many decades, have more recently been considered as a potential feed source (Musyoka et al., 2018).

Earthworms as feed have been studied in various forms (live, dry, liquid, or meal) for frogs (Latsamy \& Preston 2007), poultry (Rezaeipour et al., 2014; Bahadori et al., 2015), fish (Parachanna obscura, Vodounnou et al., 2016; Salmo gairdneri, Staffor and Tacon, 1985), freshwater shrimp (Yaqub, 1997; Langer et al., 2011; Chiu et al., 2016) and most recently shrimp (Liu et al., 2008; Beksarı, 2017; Kumlu et al., 2018). Earthworms can be abundantly and easily produced on decaying and cheap organic agricultural by-products (Chauhan et al., 2010; Fairchield et al., 2017). The method of their production not requiring expensive 
tools nor sophisticated equipment. The earthworms, are cultivated for vermicompost in many countries, are considered as a by-product derived from waste management. However, they are a good sources of high quality protein, essential amino acids and minerals (Stafford \& Tacon 1985; Paoletti et al., 2003; Istiqomah et al., 2009; Musyoka et al., 2019). There are reports that the biological value of earthworm protein is similar to that of FM and containing high proportions of essential amino acids (Tacon et al., 1983; Tacon \& Metian, 2009; NRC, 2011). However, a limiting factor characterising the overall nutritional quality of earthworm is that they are a poor source of the omega3 long chain polyunsaturated fatty acids ( $n-3$ LC-PUFA), and in particular of docosahexaenoic acid (DHA; 22:6n3) (Liu et al., 2008; Kumlu et al., 2018). Nevertheless, n3 LC-PUFAs, such as DHA and eicosapentaenoic acid (EPA; 20:5n-3), are well known to be biologically important (Leger \& Sorgeloos, 1992). This is particularly important because most of the currently farmed marine animals cannot, or have limited capacity to, bioconvert polyunsaturated fatty acids (PUFA) with 18 atoms of carbons (typically found in most animal and vegetable raw materials of terrestrial origin; Turchini et al., 2009), into LC-PUFA (Castro et al., 2016). Consequently, n-3 LCPUFA have to be present in aquaculture feed for proper animal growth, health and development (Kanazawa et al., 1979a,b; Sargent et al., 1995; Arts et al., 2001; Parrillo et al., 2017). For example, in shrimp, LC-PUFA deficiency has been found to reduce growth rate and stress resistance and increase mortality (Mutti et al., 2017).

In general, inadequate contents of essential fatty acids ( $n-3$ LC-PUFA) in the diet rise to important physiological alterations such as poor feed intake and growth, abnormal pigmentation, along with reduce fecundity, fertilization rates and embryo deformities. The nutritional importance of these fatty acids is even greater in some specific physiological and developmental stages of aquatic animal, such as early larval/post-larval stages or freshwater/marine broodstock, when often, live feed are required and utilised. However, most prey organisms commonly utilised as live feed, such as rotifers and Artemia, (Takeuchi et al., 1999; Suprayudi et al., 2004), nematodes (Kumlu et al., 1998; Honnens et al., 2014), marine worms (Klinchoedchue et al., 2011; Fairchild et al., 2017) and fairy shrimps (Velu \& Munuswamy, 2004) do not contain sufficient amount of DHA. Accordingly, they are typically enriched with n-3 LC-PUFA prior to be used as feed for farmed fish or crustaceans (Watanabe, 1993; Sorgeloos et al., 2001).

Studies on the possible n-3 LC-PUFA enrichment of worms are currently limited. On the one hand, Klinchoedchue et al. (2011) found that, in the marine sandworm (Perinereis nuntia), a diet containing high level of FO (16.7\%) resulted in an increase in arachidonic acid (ARA, 20:4n-6) and EPA, but DHA levels were unaffected. These authors concluded that sandworms should be fed for at least 26 days on an enriched diet in order to effectively change their fatty acid profiles. On the other hand, and unlike its marine counterparts, some promising results have been achieved in the terrestrial red earthworm Eisenia fetida. Beksarı (2017), and Kumlu et al. (2018) have proven that LCPUFAcontents of the REW can easily be elevated by up to 9 to 16 -fold compared to control animals by using a commercial enrichment solution within as short (12-96 h) period of time. These initial pioneering evidences have opened up a new promising area for the earthworms to be used as feed source for fish. Hence, research is warranted to enrichment methodologies, assessment of alternative low-cost enrichment mediums, and then testing the enriched worms on various farmed fish and crustaceans as well as other farmed animals, are now warranted.

Therefore, this study aimed at 1) testing the potentials of the direct use of fish oil as a readily available, and relatively less expensive when compared to commercial enrichment solutions, medium to enrich earthworm in their n-3 LC-PUFA and in particular DHA content, 2) assessing the potential of DHA fortification of earthworms via FO inclusion in their culture compost. This study was, ultimately, conceived to explore if this relatively low-cost medium could have been as efficient as the more expensive commercial products available on the market. If successful, this will provide the necessary impetus, and confidence, for possible future trials where even cheaper n-3 LC-PUFA sources, such as fish byproducts' oils, could be utilised to enrich the overall nutritional value of red earthworms, for their potential utilisation as aquaculture feed.

\section{Materials and Methods}

\section{General Culture Conditions}

This study was carried out in the Mariculture R\&D Centre of the Faculty of Fisheries of Cukurova University, Adana - Turkey. The red earthworms (Eisenia fetida) used in the experiment were obtained from a commercial producer (Argesol Tarım Hayvancılık San. Tic. Ltd., Balıkesir - Turkey). After transport, earthworms were cultured using standard procedures in three plastic tanks $(0.6 \times 3 \times 0.5 \mathrm{~m})$ for several weeks ( 3 weeks) before being used in the experiment. Briefly, in this standard procedure, the compost material used in the culture of the earthworms consisted of a blend of fermented cow manure $(50 \%)$, horse manure $(25 \%)$, and tea waste (25\%) for all the stock and culturing vessels. All the compost material mixture was fermented (mixed and sprayed with water daily) for two weeks before being used. Prior to the experiment, sufficient quantity of earthworms (initial weight $0.25 \pm 0.01 \mathrm{~g}$ ) were manually collected from the stock tanks, separated from any residual compost material, individually counted and then placed into test containers. Each enrichment group was assigned to three replicates. The experiment was 
conducted indoor in an air-conditioned room in three 1 $\mathrm{L}$, flat bottom plastic containers, allocated to each of the four treatment groups. Compost temperature, $\mathrm{pH}$ and moisture were measured daily during the experiment by hand-held digital thermometer, $\mathrm{pH}$-meter and moisture meter, respectively. The moisture of the compost in the culture containers was maintained at levels between 70 and $75 \%$ by spraying water twice daily in the morning and in the late afternoon, as needed. Temperature was maintained at constant $26^{\circ} \mathrm{C}$, and $\mathrm{pH}$ remained between 7.1 and 7.3 throughout the experiment.

The fish oil (FO) used in the experiment was anchovy oil (Engraulis encrasicolus) supplied by Sibal Inc., Sinop (Turkey). Four doses of FO (0, 2.5, 5 and $10 \mathrm{~g}$ FO/100 g compost), at four different period of enrichment exposures (0, 24, 48 and 96 h), were assessed to see the combined effects of the two variables (dose and time) on the LC-PUFA, and in particular DHA, content of the earthworms. The experimental treatments were named accordingly, as: 0 FO (Control: $100 \mathrm{~g}$ bedding $+100 \mathrm{~g}$ compost $+0 \mathrm{~g}$ fish oil), $2.5 \mathrm{FO}$ (100 g bedding $+100 \mathrm{~g}$ compost $+5 \mathrm{~g}$ fish oil), 5 FO (100 g bedding +100 g compost $+5 \mathrm{~g}$ fish oil), and 10 FO (100 g bedding $+100 \mathrm{~g}$ compost $+10 \mathrm{~g}$ fish oil). Three 1-L flat bottom plastic containers, each drilled with 15 holes $(0.5 \mathrm{~mm})$, were allocated to each experimental group. The bedding material used was composed of exhausted (used) composting material (blend of fermented cow manure (50\%), horse manure $(25 \%)$, and tea waste $(25 \%)$ that had been under use for 6 weeks in the culture of the earthworms' stock tanks. Before stocking the earthworms into the experimental containers, $100 \mathrm{~g}$ of bedding material was placed into each culture container (1-L). Then, the mixture of $100 \mathrm{~g}$ of compost + the corresponding level of FO was placed on top of the bedding material. Finally, 300 adult worms were stocked into each culture container and the samplings were implemented at 0, 24, 48 and $96 \mathrm{~h}$ of the culture period. All containers were covered with dark sheeting throughout the experimental period. At each sampling time, 50 adult worms were sampled, washed thoroughly with freshwater until no residue of compost was visible, and placed into $20-\mathrm{mL}$ plastic tubes and then immediately frozen at $-20^{\circ} \mathrm{C}$ until subsequent analysis.

\section{Lipid and Fatty Acid Analyses}

After thawing the stored frozen samples, about $4 \mathrm{~g}$ of samples (15-20 worms) per replicate were ground to a homogeneous consistency using a centrifugal mill fitted with a $0.25 \mathrm{~mm}$ screen, before analysis. Lipids were extracted according to the procedure of Folch et al. (1957). Fatty acid methyl esters were prepared according to Metcalfe \& Schmitz (1961) and then separated and analysed as described previously by Czesny and Dabrowski (1998), with minor modifications (the oven temperature program was: $190^{\circ} \mathrm{C}$ for $35 \mathrm{~min}$, then increasing at $30^{\circ} \mathrm{C}$ per min up to $220^{\circ} \mathrm{C}$, and then maintained for $5 \mathrm{~min}$ ). Briefly, the fatty acid methyl esters obtained were separated by gas chromatography (Agilent 6820A; Agilent Technologies, Santa Clara, California, USA), equipped with a flame ionization detector and fitted with a DB 23 capillary column $(60 \mathrm{~m}$, $0.25 \mathrm{~mm}$ i.d. and $0.25 \mu \mathrm{m}$; Agilent Technologies, Santa Clara, California, USA). The carrier gas was hydrogen at

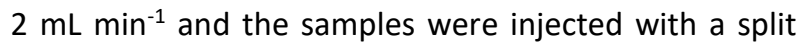
ratio of 30:1. Fatty acids were identified by comparing their retention times to that of a standard mix of fatty acids (Supelco 37 component FAME mix; Sigma-Aldrich Pty. Ltd., Interlab Inc., Istanbul, Turkey). When external fatty acid standards were not available, identification was confirmed by additional GC/MS analysis (Agilent 6890 GC-5973 MS, Agilent Technologies, Wilmington, $D E$, USA). Resulting peak areas were corrected by the

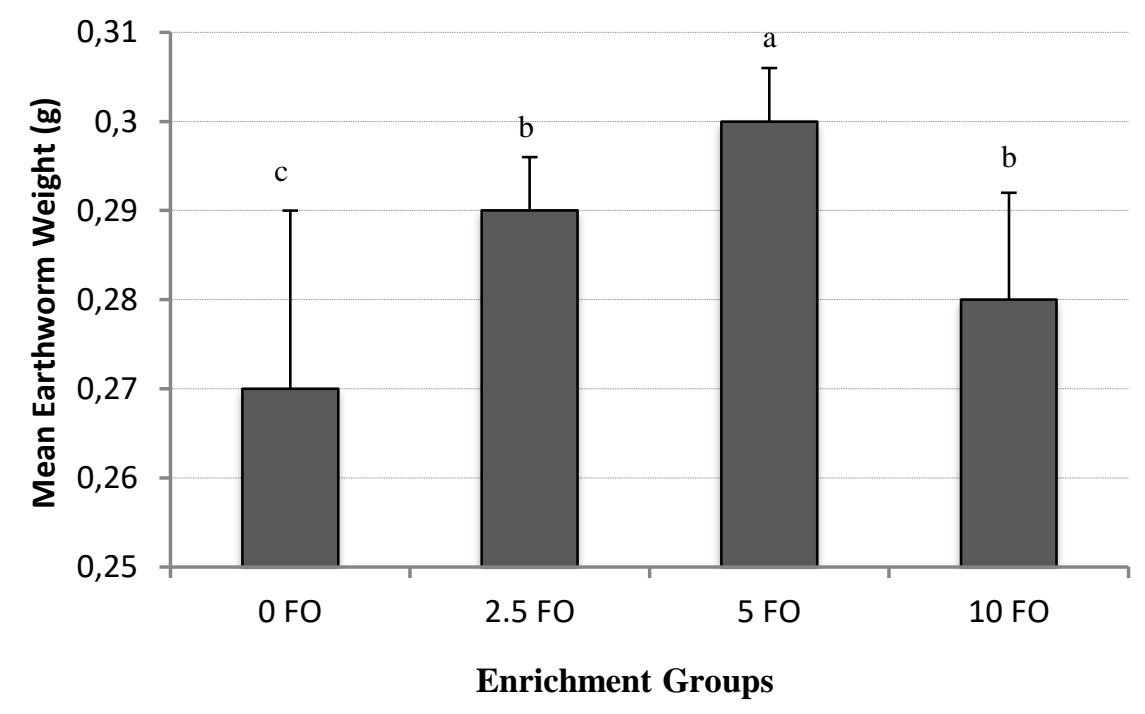

Figure 1. Mean weight of the earthworms Eisenia fetida enriched for up to 96 -h with different doses of fish oil $(0,2.5,5.0$ and $10 \%$ FO). Each datapoint represents mean $\pm s d(m o l \%, n=3)$. Dots marked with different letters for each enrichment-period are significantlv different from each other $(\mathrm{P}<0.05)$. 
theoretical relative FID response factors and for methyl transformation, and then quantified and reported as mole percentage (mol\%), relative to total fatty acids.

\section{Statistical Analyses}

Following confirmation of normality and homogeneity of variance by Levene's test, data was analysed by two-way analysis of variance (two-way ANOVA), assessing the effects of enrichment dose, enrichment time, and interactions between the two. Where significant differences $(P<0.05)$ were detected, a Duncan post hoc test was computed for identifying any significant difference among the Treatments groups and determine homogenous subset based on the enriching dose at a fixed time or vice versa. All computations were performed using SPSS 20.0 (SPSS Inc., Chicago, IL, USA).

\section{Results}

During enrichment period, there was no significant difference in survival rate of the groups $(P>0.05)$. Thus, in the present study, there were not significant effects of the enrichment protocols (dose and time) applied on survival rate of the REW. However, growth rate of the REW was significantly affected by enrichment dose of FO in the compost. As shown in Fig. 1, the best growth was found in the 5 FO group at $96 \mathrm{~h}(\mathrm{P}<0.05)$.
The fatty acid composition of the unenriched earthworms and that of the FO used for the enrichment trial is reported (Table 1 and Table 2). In general, SFA content of FO (32.91 mol\%) was higher than that recorded in the worms (21.42 mol\%). Specifically, 14:0 (4.96 mol\%) and 16:0 (20.22 mol\%) levels found in FO were much higher than in the worms ( 0.87 to 2.46 mol\%), whereas 12:0 was showing an opposite trend and it was higher in the worms (1.69 mol\%) compared to FO (0.09 mol\%). MUFA were higher in FO (31.47 mol\%) compared to worms (26.72 mol\%), and this was primarily driven by a remarkable difference in the content of 18:1n-9, which was 18.21 mol\% in FO and only $2.87 \mathrm{~mol} \%$ in the worms. The lower SFA and MUFA content of worms, compared to FO, resulted in higher content of PUFA, with PUFA level in worms being 51.87 mol\%, versus 35.62 mol\% in FO. The worms were found to be richer than FO in terms of $18: 2 n-6$ (10.21 vs 3.53 mol\%), 18: $3 n-3$ (11.89 vs 1.52 mol\%), 20:2n-6 (2.56 vs 0.39 mol\%), $20: 3 n-3$ (10.00 vs $2.61 \%$ ) and interestingly also $20: 5 n-3$ (EPA; 9.06 vs 7.80 mol\%). On the contrary, FO was remarkably richer (over 6-fold) in 22:6n-3 (DHA; $15.40 \mathrm{~mol} \%$ ) compared to worms (2.27 mol\%). Although the worms contained higher amounts of PUFA their DHA, DHA / EPA ratio and n-3 / n-6 ratio were all remarkably lower than those observed in FO.

Overall, two-way ANOVA results showed significant differences in almost all FA and FA classes,

Table 1. Fatty acids (FA, mol\%) composition of the earthworms (Eisenia fetida) before enrichment, and the fish oil (FO) used for the enrichment experiment. Each value is a mean of three repeated analytical assesments.

\begin{tabular}{|c|c|c|}
\hline Fatty Acids & Earthworms & Fish Oil \\
\hline $12: 0$ & 1.69 & 0.09 \\
\hline $14: 0$ & 0.87 & 4.96 \\
\hline $16: 0$ & 2.46 & 20.22 \\
\hline Other SFA $A^{1}$ & 16.4 & 7.67 \\
\hline SFA $^{2}$ & 21.42 & 32.91 \\
\hline $16: 1 n-7$ & 0.70 & 4.93 \\
\hline $18: 1 n-7$ & 8.53 & 2.75 \\
\hline $18: 1 n-9$ & 2.87 & 18.21 \\
\hline Other MUFA & 14.62 & 5.58 \\
\hline MUFA $^{4}$ & 26.72 & 31.47 \\
\hline $18: 2 n-6$ & 10.21 & 3.53 \\
\hline $20: 4 n-6$ & 1.24 & 0.75 \\
\hline Other $n-6$ PUFA & 4.7 & 2.37 \\
\hline n-6 PUFA 6 & 16.15 & 6.65 \\
\hline $18: 3 n-3$ & 11.89 & 1.52 \\
\hline $20: 5 n-3$ (EPA) & 9.06 & 7.80 \\
\hline $22: 6 n-3(D H A)$ & 2.27 & 15.40 \\
\hline Other $n-3$ PUFA & 1.15 & 1.12 \\
\hline$n-3$ PUFA $^{8}$ & 34.37 & 28.45 \\
\hline PUFA $^{9}$ & 51.87 & 35.62 \\
\hline n-3/n-6 PUFA & 2.13 & 4.28 \\
\hline DHA+EPA & 11.30 & 23.20 \\
\hline DHA/EPA & 0.25 & 1.97 \\
\hline
\end{tabular}

${ }^{1}$ Sum of $10: 0,13: 0,15: 0,17: 0,18: 0$ and $24: 0$

${ }^{2}$ Sum of all Saturated Fatty Acids

${ }^{3}$ Sum of $10: 1,12: 1 n-3,12: 1 n-5,14: 1,15: 1,17: 1 n-7,20: 1 n-9,20: 1 n-11,22: 1 n-9$ and $24: 1 n-9$

${ }^{4}$ Sum of all Monounsaturated Fatty Acids

${ }^{5}$ Sum of $18: 3 n-6,20: 2 n-6,20: 3 n-6$

${ }^{6}$ Sum of all omega- 6 Polyunsaturated Fatty Acids

${ }^{7}$ Sum of $20: 3 n-3$ and $22: 5 n-3$

${ }^{8}$ Sum of all omega-3 Polyunsaturated Fatty Acids

${ }^{9}$ Sum of all Polyunsaturated Fatty Acids 
Table 2. Fatty acids (FA, mol\%) composition of the earthworms (Eisenia fetida) enriched for $0,24,48$ and $96 \mathrm{~h}$ in four doses of fish oil (FO) $(0,2.5,5.0$ and $10 \mathrm{~g}$ FO per $100 \mathrm{~g}$ compost) for up to 96 -h enrichment period. Each value is a mean $(\mathrm{n}=3) \pm \mathrm{standard}$ deviation $(\mathrm{sd})$.

\begin{tabular}{|c|c|c|c|c|c|}
\hline \multirow[b]{2}{*}{$\begin{array}{l}\text { Fatty Acids } \\
\text { (Mol\%)* }\end{array}$} & \multicolumn{4}{|c|}{ Enrichment Dose (g / 100 g compost) } & \multirow[b]{2}{*}{$P$} \\
\hline & $0 \mathrm{FO}$ (Control) & $2.5 \mathrm{FO}$ & $5 \mathrm{FO}$ & $10 \mathrm{FO}$ & \\
\hline \multicolumn{6}{|l|}{ O h Enrichment } \\
\hline $12: 0$ & $1.69 \pm 0.17^{\Upsilon}$ & $1.69 \pm 0.17^{\mathrm{ar}}$ & $1.69 \pm 0.17^{\mathrm{aY}}$ & $1.69 \pm 0.17^{\mathrm{ar}}$ & 1.000 \\
\hline 14:0 & $0.87 \pm 0.48^{\mathrm{aY}}$ & $0.87 \pm 0.48^{\mathrm{aY}}$ & $0.87 \pm 0.48^{\mathrm{aY}}$ & $0.87 \pm 0.48^{\mathrm{aY}}$ & 1.000 \\
\hline $16: 0$ & $2.46 \pm 0.17^{\mathrm{aW}}$ & $2.46 \pm 0.17 \mathrm{aW}$ & $2.46 \pm 0.17^{\mathrm{aW}}$ & $2.46 \pm 0.17^{\mathrm{aW}}$ & 1.000 \\
\hline Other SFA ${ }^{1}$ & $16.4 \pm 0.59 \mathrm{aw}$ & $16.4 \pm 0.59 \mathrm{aW}$ & $16.4 \pm 0.59^{\mathrm{aW}}$ & $16.4 \pm 0.59^{\mathrm{aW}}$ & 1.000 \\
\hline SFA $^{2}$ & $21.42 \pm 0.64^{\mathrm{aW}}$ & $21.42 \pm 0.64^{\mathrm{aW}}$ & $21.42 \pm 0.64^{\mathrm{aW}}$ & $21.42 \pm 0.64^{\mathrm{aW}}$ & 1.000 \\
\hline $16: 1 n-7$ & $0.70 \pm 0.01^{\mathrm{aY}}$ & $0.70 \pm 0.01^{\mathrm{aY}}$ & $0.70 \pm 0.01^{\mathrm{aY}}$ & $0.70 \pm 0.01^{\mathrm{aY}}$ & 1.000 \\
\hline $18: 1 n-7$ & $8.53 \pm 0.77 \mathrm{aW}$ & $8.53 \pm 0.77 \mathrm{aW}$ & $8.53 \pm 0.77$ aw & $8.53 \pm 0.77 \mathrm{aW}$ & 1.000 \\
\hline $18: 1 n-9$ & $2.87 \pm 0.12 \mathrm{aY}$ & $2.87 \pm 0.12 \mathrm{ar}$ & $2.87 \pm 0.12 \mathrm{aY}$ & $2.87 \pm 0.12 \mathrm{aY}$ & 1.000 \\
\hline Other MUFA ${ }^{3}$ & $14.62 \pm 1.06 \mathrm{aW}$ & $14.62 \pm 1.06 \mathrm{aW}$ & $14.62 \pm 1.06^{\mathrm{aW}}$ & $14.62 \pm 1.06^{\mathrm{aW}}$ & 1.000 \\
\hline MUFA $^{4}$ & $26.72 \pm 0.65 \mathrm{aW}$ & $26.72 \pm 0.65 \mathrm{aW}$ & $26.72 \pm 0.65 \mathrm{aW}$ & $26.72 \pm 0.65 \mathrm{aW}$ & 1.000 \\
\hline $18: 2 n-6$ & $10.21 \pm 0.45^{\mathrm{ax}}$ & $10.21 \pm 0.45^{\mathrm{ax}}$ & $10.21 \pm 0.45^{a x}$ & $10.21 \pm 0.45^{\mathrm{ax}}$ & 1.000 \\
\hline $20: 4 n-6$ & $1.24 \pm 0.18 \mathrm{aW}$ & $1.24 \pm 0.18 \mathrm{aW}$ & $1.24 \pm 0.18 \mathrm{aW}$ & $1.24 \pm 0.18^{\mathrm{aW}}$ & 1.000 \\
\hline Other $n-6$ PUFA $^{5}$ & $4.72 \pm 0.05 \mathrm{aW}$ & $4.72 \pm 0.05 \mathrm{aW}$ & $4.72 \pm 0.05 \mathrm{aW}$ & $4.72 \pm 0.05 \mathrm{aW}$ & 1.000 \\
\hline n-6 PUFA 6 & $16.15 \pm 0.46 \mathrm{aW}$ & $16.15 \pm 0.46 \mathrm{aW}$ & $16.15 \pm 0.46^{\mathrm{aW}}$ & $16.15 \pm 0.46 \mathrm{aW}$ & 1.000 \\
\hline $18: 3 n-3$ & $11.89 \pm 0.67 \mathrm{aW}$ & $11.89 \pm 0.67 \mathrm{aW}$ & $11.89 \pm 0.67^{\mathrm{aW}}$ & $11.89 \pm 0.67^{\mathrm{aW}}$ & 1.000 \\
\hline 20:5n-3 (EPA) & $9.06 \pm 0.31 \mathrm{az}$ & $9.06 \pm 0.31 \mathrm{az}$ & $9.06 \pm 0.31 \mathrm{az}$ & $9.06 \pm 0.31 \mathrm{az}$ & 1.000 \\
\hline $22: 6 n-3(D H A)$ & $2.27 \pm 0.21 \mathrm{az}$ & $2.27 \pm 0.21 \mathrm{az}$ & $2.27 \pm 0.21 \mathrm{az}$ & $2.27 \pm 0.21 \mathrm{az}$ & 1.000 \\
\hline Other $n-3$ PUFA ${ }^{7}$ & $1.15 \pm 0.14^{\mathrm{ax}}$ & $1.15 \pm 0.14^{\mathrm{ax}}$ & $1.15 \pm 0.14 \mathrm{ax}$ & $1.15 \pm 0.14^{\mathrm{ax}}$ & 1.000 \\
\hline n-3 PUFA ${ }^{8}$ & $34.37 \pm 0.76 \mathrm{ar}$ & $34.37 \pm 0.76 \mathrm{aY}$ & $34.37 \pm 0.76 \mathrm{aY}$ & $34.37 \pm 0.76 \mathrm{aY}$ & 1.000 \\
\hline PUFA $^{9}$ & $51.87 \pm 0.45^{\mathrm{a}}$ & $51.87 \pm 0.45^{\mathrm{a} Y}$ & $51.87 \pm 0.45^{\mathrm{a} Y}$ & $51.87 \pm 0.45^{\mathrm{aY}}$ & 1.000 \\
\hline n-3/n-6 PUFA & $2.13 \pm 0.03 \mathrm{az}$ & $2.13 \pm 0.03 \mathrm{az}$ & $2.13 \pm 0.03 \mathrm{az}$ & $2.13 \pm 0.03 \mathrm{az}$ & 1.000 \\
\hline DHA+EPA & $11.30 \pm 0.49 \mathrm{az}$ & $11.30 \pm 0.49 \mathrm{az}$ & $11.30 \pm 0.49 \mathrm{az}$ & $11.30 \pm 0.49 \mathrm{az}$ & 1.000 \\
\hline DHA/EPA & $0.25 \pm 0.02 \mathrm{az}$ & $0.25 \pm 0.02 \mathrm{az}$ & $0.25 \pm 0.02 \mathrm{az}$ & $0.25 \pm 0.02 \mathrm{az}$ & 1.000 \\
\hline \multicolumn{6}{|l|}{$24 \mathrm{~h}$ Enrichment } \\
\hline $12: 0$ & $2.71 \pm 0.06^{\mathrm{ax}}$ & $2.74 \pm 0.07^{\mathrm{ax}}$ & $2.23 \pm 0.06^{b x}$ & $2.29 \pm 0.06^{b x}$ & 0.000 \\
\hline $14: 0$ & $1.34 \pm 0.02 c x$ & $1.57 \pm 0.01 \mathrm{bx}$ & $1.25 \pm 0.02 c x$ & $1.94 \pm 0.15^{\mathrm{ax}}$ & 0.000 \\
\hline $16: 0$ & $2.32 \pm 0.05 \mathrm{cz}$ & $2.55 \pm 0.01 \mathrm{bz}$ & $2.17 \pm 0.03 \mathrm{dz}$ & $2.82 \pm 0.02 \mathrm{az}$ & 0.000 \\
\hline Other SFA & $13.08 \pm 2.48 \mathrm{ax}$ & $12.13 \pm 2.21^{b x}$ & $12.05 \pm 2.65^{b x}$ & $9.71 \pm 2.15^{c x}$ & 0.000 \\
\hline SFA & $19.08 \pm 2.00 \mathrm{az}$ & $18.99 \pm 1.78^{a z}$ & $17.71 \pm 2.11^{b z}$ & $16.78 \pm 1.75^{b z}$ & 0.000 \\
\hline $16: 1 n-7$ & $0.58 \pm 0.08 \mathrm{bY}$ & $0.68 \pm 0.06^{b Y}$ & $0.59 \pm 0.05$ bY & $1.05 \pm 0.01^{\mathrm{aY}}$ & 0.000 \\
\hline $18: 1 n-7$ & $7.32 \pm 0.12^{b x}$ & $6.99 \pm 0.05^{c x}$ & $7.65 \pm 0.08$ ax & $6.30 \pm 0.05 \mathrm{dx}$ & 0.000 \\
\hline $18: 1 n-9$ & $2.41 \pm 0.04 \mathrm{cz}$ & $3.00 \pm 0.02 \mathrm{bz}$ & $2.28 \pm 0.06 \mathrm{dz}$ & $3.15 \pm 0.01 \mathrm{az}$ & 0.000 \\
\hline Other MUFA & $16.23 \pm 1.65 \mathrm{aw}$ & $16.26 \pm 1.69 \mathrm{aW}$ & $11.28 \pm 1.35^{b W}$ & $12.84 \pm 1.00 \mathrm{bW}$ & 0.000 \\
\hline MUFA & $26.24 \pm 2.19 \mathrm{aX}$ & $26.92 \pm 2.13 \mathrm{ax}$ & $21.80 \pm 2.28 c x$ & $23.54 \pm 1.73^{b x}$ & 0.020 \\
\hline $18: 2 n-6$ & $11.27 \pm 0.22^{b W}$ & $10.77 \pm 0.01^{\mathrm{aW}}$ & $11.97 \pm 0.21 \mathrm{cW}$ & $9.04 \pm 0.02 \mathrm{dW}$ & 0.000 \\
\hline $20: 4 n-6$ & $1.63 \pm 0.13^{a x}$ & $1.63 \pm 0.03^{a x}$ & $1.58 \pm 0.12^{\mathrm{ax}}$ & $1.32 \pm 0.02^{b x}$ & 0.005 \\
\hline Other $n-6$ PUFA & $4.21 \pm 1.01 \mathrm{bx}$ & $4.04 \pm 1.10^{b x}$ & $4.30 \pm 1.04 \mathrm{ax}$ & $3.23 \pm 0.72 c x$ & 0.000 \\
\hline n-6 PUFA & $17.12 \pm 6.86^{\mathrm{bW}}$ & $16.58 \pm 4.22 \mathrm{cW}$ & $17.97 \pm 4.74 \mathrm{aW}$ & $13.64 \pm 3.57 \mathrm{aW}$ & 0.000 \\
\hline $18: 3 n-3$ & $9.21 \pm 0.33 b x$ & $9.33 \pm 0.34 b x$ & $10.93 \pm 0.11^{\mathrm{ax}}$ & $7.45 \pm 0.38 \mathrm{cx}$ & 0.000 \\
\hline $20: 5 n-3$ (EPA) & $13.58 \pm 0.20^{\mathrm{bW}}$ & $13.47 \pm 0.14^{\mathrm{bW}}$ & $14.81 \pm 0.07 \mathrm{aw}$ & $13.33 \pm 0.11^{\mathrm{bw}}$ & 0.000 \\
\hline $22: 6 n-3(D H A)$ & $0.92 \pm 0.05^{b W}$ & $1.12 \pm 0.10^{\mathrm{bW}}$ & $0.96 \pm 0.03 \mathrm{bW}$ & $14.01 \pm 0.66 \mathrm{aW}$ & 0.000 \\
\hline Other $n-3$ PUFA & $12.28 \pm 7.10^{\mathrm{bW}}$ & $12.19 \pm 7.06 \mathrm{bW}$ & $14.10 \pm 7.95 \mathrm{aw}$ & $10.77 \pm 5.55 \mathrm{cw}$ & 0.000 \\
\hline n-3 PUFA & $35.99 \pm 5.85^{c x}$ & $36.11 \pm 5.77 \mathrm{bcx}$ & $40.80 \pm 6.51 \mathrm{abx}$ & $45.55 \pm 5.08 \mathrm{ax}$ & 0.004 \\
\hline PUFA & $54.36 \pm 5.16 \mathrm{bW}$ & $53.64 \pm 5.10 \mathrm{cW}$ & $59.64 \pm 5.78 \mathrm{aW}$ & $59.19 \pm 5.33$ aW & 0.000 \\
\hline n-3/n-6 PUFA & $2.10 \pm 0.03^{c \gamma}$ & $2.18 \pm 0.15^{\mathrm{cY}}$ & $2.27 \pm 0.12^{b Y}$ & $3.39 \pm 0.22 \mathrm{ar}$ & 0.000 \\
\hline $\mathrm{DHA}+\mathrm{EPA}$ & $14.50 \pm 0.18^{b Y}$ & $14.59 \pm 0.22 \mathrm{bY}$ & $15.77 \pm 0.24$ br & $27.34 \pm 0.78^{\mathrm{ar}}$ & 0.000 \\
\hline DHA/EPA & $0.07 \pm 0.01 \mathrm{bY}$ & $0.08 \pm 0.01^{b Y}$ & $0.06 \pm 0.02^{b Y}$ & $1.05 \pm 0.04$ bY & 0.000 \\
\hline \multicolumn{6}{|l|}{$48 \mathrm{~h}$ Enrichment } \\
\hline $12: 0$ & $2.64 \pm 0.21 \mathrm{cw}$ & $6.65 \pm 0.14 \mathrm{aW}$ & $2.32 \pm 0.02 \mathrm{dW}$ & $3.63 \pm 0.01 \mathrm{bw}$ & 0.000 \\
\hline $14: 0$ & $0.89 \pm 0.59 \mathrm{dW}$ & $4.67 \pm 0.09 \mathrm{aW}$ & $1.76 \pm 0.14 \mathrm{cW}$ & $3.01 \pm 0.03 \mathrm{bW}$ & 0.000 \\
\hline $16: 0$ & $1.87 \pm 0.08 \mathrm{dx}$ & $4.76 \pm 0.07 \mathrm{ax}$ & $2.71 \pm 0.02 \mathrm{cx}$ & $3.42 \pm 0.02^{b x}$ & 0.000 \\
\hline Other SFA & $14.29 \pm 3.21^{\mathrm{aY}}$ & $9.09 \pm 2.09 \mathrm{cr}$ & $10.28 \pm 2.35^{b Y}$ & $9.33 \pm 1.91^{\mathrm{cY}}$ & 0.000 \\
\hline SFA & $19.70 \pm 2.60 \mathrm{bY}$ & $25.19 \pm 2.60 \mathrm{aY}$ & $17.06 \pm 1.89 c \gamma$ & $19.38 \pm 1.76$ bY & 0.000 \\
\hline
\end{tabular}


Table 2. Continued

$16: 1 \mathrm{n}-7$

18:1n-7

18:1n-9

Other MUFA

MUFA

$18: 2 n-6$

20:4n-6

Other $n-6$ PUFA

n-6 PUFA

18:3n-3

20:5n-3 (EPA)

22:6n-3 (DHA)

Other $n-3$ PUFA

n-3 PUFA

\section{PUFA}

n-3/n-6 PUFA

DHA+EPA

DHA/EPA

\section{$96 \mathrm{~h}$ Enrichment}

$12: 0$
$14: 0$
$16: 0$
Other SFA

\section{SFA}

16:1n-7

18:1n-7

18:1n-9

Other MUFA

MUFA

$18: 2 n-6$

20:4n-6

Other $n-6$ PUFA

n-6 PUFA

18:3n-3

20:5n-3 (EPA)

22:6n-3 (DHA)

Other n-3 PUFA

n-3 PUFA

\section{PUFA}

$n-3 / n-6$ PUFA

DHA+EPA

DHA/EPA

n.d..$^{\mathrm{cx}}$
$7.89 \pm 0.28^{\mathrm{ar}}$
$4.05 \pm 0.62^{\mathrm{bw}}$
$16.49 \pm 1.38^{\mathrm{ax}}$
$\mathbf{2 8 . 4 3} \pm 2.24^{\mathrm{ax}}$
$10.53 \pm 0.39^{\mathrm{ar}}$
$0.90 \pm 0.08^{\mathrm{cx}}$
$4.14 \pm 0.94^{\mathrm{ar}}$
$15.57 \pm 4.21^{\mathrm{ax}}$
$9.88 \pm 0.39^{\mathrm{az}}$
$11.46 \pm 0.61^{\mathrm{br}}$
$0.46 \pm 0.11^{\mathrm{cr}}$
$12.28 \pm 6.55^{\mathrm{ar}}$
$34.08 \pm 5.36^{\mathrm{dW}}$
$\mathbf{5 0 . 8 7} \pm 4.21^{\mathrm{cx}}$
$2.19 \pm 0.54^{\mathrm{cW}}$
$11.92 \pm 0.68^{\mathrm{bW}}$
$0.04 \pm 0.01^{\mathrm{cW}}$

$2.71 \pm 0.17$ bx

$0.88 \pm 0.56 \mathrm{dW}$

$1.95 \pm 0.05^{\mathrm{dY}}$

$14.63 \pm 3.27$ ax

$20.17 \pm 2.64$ bx

$0.73 \pm 0.03 \mathrm{dW}$

$8.50 \pm 0.90^{\mathrm{ar}}$

$2.52 \pm 0.22 \mathrm{dX}$

$15.18 \pm 1.40 \mathrm{ax}$

26.92 \pm 2.36 ax

$10.94 \pm 0.51^{\mathrm{az}}$

$0.91 \pm 0.10^{c \mathrm{x}}$

$3.96 \pm 0.90$ aY

$15.81 \pm 4.40^{\mathrm{ax}}$

$9.82 \pm 0.43$ aY

$11.85 \pm 0.82 \mathrm{bx}$

$1.74 \pm 0.78 \mathrm{dx}$

$12.49 \pm 6.48$ ax

$35.89 \pm 5.05 \mathrm{cwx}$

\section{$52.91 \pm 4.84 c x$}

$2.27 \pm 0.13$ ax

$13.59 \pm 1.43 \mathrm{dx}$

$0.15 \pm 0.03 \mathrm{dx}$

\begin{abstract}
n.d. $c x$
$6.43 \pm 0.14$ by

$5.13 \pm 0.08$ aw

$9.60 \pm 0.98 \mathrm{cx}$

$23.30 \pm 2.03 \mathrm{cbx}$

$6.11 \pm 0.07 \mathrm{dY}$

$1.16 \pm 0.02 \mathrm{bx}$

$2.95 \pm 0.63$ by

$10.22 \pm 2.32 \mathrm{bx}$

$5.21 \pm 0.04 \mathrm{cz}$

$11.60 \pm 0.11^{\mathrm{br}}$

$15.55 \pm 1.07$ ar

$7.51 \pm 3.61 \mathrm{cr}$

$39.87 \pm 5.63 \mathrm{cW}$

$50.66 \pm 5.04 c x$

$3.90 \pm 0.12 \mathrm{aW}$

$27.15 \pm 0.95 \mathrm{aW}$

$1.34 \pm 0.04$ aw
\end{abstract}

$2.55 \pm 0.04 \mathrm{bx}$
$1.72 \pm 0.04 \mathrm{cw}$
$2.75 \pm 0.03^{\mathrm{cr}}$
$10.82 \pm 2.07^{\mathrm{bx}}$

$17.83 \pm 1.68 \mathrm{cx}$

$1.03 \pm 0.03 \mathrm{cW}$

$6.28 \pm 0.11$ by

$2.89 \pm 0.20^{\mathrm{cx}}$

$11.55 \pm 1.37 \mathrm{bcx}$

21.77 $\pm 1.88 \mathrm{dx}$

$9.35 \pm 0.19^{b z}$

$1.52 \pm 0.05^{a x}$

$3.66 \pm 0.84 \mathrm{aby}$

$14.52 \pm 3.65^{b x}$

$7.50 \pm 0.12^{\mathrm{bY}}$

$13.70 \pm 0.13$ ax

$12.58 \pm 0.46^{\mathrm{ax}}$

$10.37 \pm 5.58 \mathrm{bx}$

$44.53 \pm 4.91 \mathrm{awx}$

\section{$59.87 \pm 5.13$ ax}

$3.07 \pm 0.08 \mathrm{ax}$

$26.28 \pm 0.37 \mathrm{ax}$

$0.92 \pm 0.02$ ax

\begin{tabular}{|c|c|}
\hline $2.15 \pm 0.05^{\mathrm{ax}}$ & $1.02 \pm 0.04^{\mathrm{bx}}$ \\
\hline $6.27 \pm 0.06^{b Y}$ & $6.19 \pm 0.06^{b Y}$ \\
\hline $3.15 \pm 0.03 \mathrm{cW}$ & $3.87 \pm 0.02 \mathrm{bW}$ \\
\hline $12.29 \pm 1.16^{\mathrm{bx}}$ & $12.27 \pm 1.19 \mathrm{bx}$ \\
\hline $22.73 \pm 1.78 c x$ & $23.82 \pm 1.81 \mathrm{bx}$ \\
\hline $9.21 \pm 0.12^{b r}$ & $8.17 \pm 0.06^{c \gamma}$ \\
\hline $1.42 \pm 0.03^{a x}$ & $1.34 \pm 0.02 \mathrm{ax}$ \\
\hline $3.46 \pm 0.79 \mathrm{bY}$ & $3.31 \pm 0.73^{b y}$ \\
\hline $14.09 \pm 3.62 \mathrm{abx}$ & $12.81 \pm 3.18^{b x}$ \\
\hline $7.11 \pm 0.09 \mathrm{bz}$ & $7.02 \pm 0.07^{b z}$ \\
\hline $13.61 \pm 0.10^{\mathrm{aY}}$ & $13.68 \pm 0.11^{\mathrm{aY}}$ \\
\hline $13.79 \pm 0.82^{b Y}$ & $12.51 \pm 0.79 \mathrm{bY}$ \\
\hline $10.36 \pm 5.64 \mathrm{bY}$ & $9.44 \pm 4.99 \mathrm{bY}$ \\
\hline $44.86 \pm 5.21 \mathrm{aW}$ & $42.65 \pm 4.97 \mathrm{bW}$ \\
\hline $59.74 \pm 5.29 a x$ & $56.15 \pm 5.00^{\mathrm{bx}}$ \\
\hline $3.18 \pm 0.08^{b W}$ & $3.33 \pm 0.07^{\mathrm{bW}}$ \\
\hline $27.40 \pm 0.72 \mathrm{aW}$ & $26.19 \pm 0.69 \mathrm{aW}$ \\
\hline
\end{tabular}

$3.63 \pm 0.02 \mathrm{bx}$

$3.09 \pm 0.02 \mathrm{bW}$

$3.40 \pm 0.76^{\mathrm{bY}}$

$9.68 \pm 1.92 \mathrm{bx}$

$19.80 \pm 1.76$ bx

$1.56 \pm 0.02 \mathrm{bW}$

$6.03 \pm 0.03$ by

$3.93 \pm 0.03 \mathrm{bx}$

$12.57 \pm 1.23 \mathrm{bx}$

24.10 $\pm 1.78 \mathrm{cx}$

$8.44 \pm 0.07^{c z}$

$1.44 \pm 0.03$ ax

$3.48 \pm 0.81$ br

$13.35 \pm 3.28 \mathrm{cx}$

$7.28 \pm 0.03 \mathrm{cr}$

$13.55 \pm 0.04$ ax

$10.99 \pm 0.44 \mathrm{bx}$

$9.57 \pm 4.91 \mathrm{cx}$

$41.39 \pm 4.60 \mathrm{bwx}$

$6.20 \pm 0.01$ ax

$4.51 \pm 0.02 \mathrm{aW}$

$5.29 \pm 0.05$ ar

$10.21 \pm 2.21^{\mathrm{bx}}$

26.20 \pm 2.55 ax

$2.17 \pm 0.02 \mathrm{aw}$

$6.66 \pm 0.05^{b y}$

$5.88 \pm 0.06$ ax

$10.78 \pm 1.04 \mathrm{cx}$

$25.48 \pm 2.16 \mathrm{dx}$

$6.91 \pm 0.18 \mathrm{dz}$

$1.27 \pm 0.02 \mathrm{bx}$

$3.33 \pm 0.73$ by

$11.51 \pm 2.62 \mathrm{dx}$

$5.75 \pm 0.09 \mathrm{dY}$

$12.40 \pm 0.16 \mathrm{bx}$

$8.87 \pm 0.59 c x$

$8.32 \pm 4.04 \mathrm{dx}$

$35.35 \pm 4.08 \mathrm{cwx}$

$55.42 \pm 4.77^{b x}$

$3.10 \pm 0.07$ ax

$24.54 \pm 0.49 \mathrm{bx}$

$0.81 \pm 0.03^{b x}$
$47.46 \pm 4.08 \mathrm{dx}$

$3.07 \pm 0.10^{\mathrm{ax}}$

$21.27 \pm 0.51$ cx

$0.72 \pm 0.04 \mathrm{bcx}$
0.000

0.000

0.000

0.000

0.000

0.006

.037

.000

0.000

0.000

0.000

0.000

.000

0.000

0.000

0.000

0.000

0.000

0.000

0.001

0.000

0.001

0.000

0.000

0.000

0.014

0.000

0.000

0.002

0.000

0.000

0.000

0.000

0.000

0.000 0.000

Different lower-case letters (abcd) indicate significant differences $(P<0.05)$ for the same fatty acids/groups among enriching times; different uppercase letters (WXYZ) indicate significant differences $(P<0.05)$ among doses for individual fatty acids/ groups $(P<0.05)$. n.d. represent not detected.

* See Table 1 for FA classes, sums and ratios descriptions.

relative to the effects of dose, time and their interactions (Table 2 and Table 3). FO-enrichment in this study led to significant increases in n-3 PUFA levels from base levels of $34.08-35.99 \mathrm{~mol} \%$ to $36.11-45.55 \mathrm{~mol} \%$ at $24 \mathrm{~h}, 39.87-44.86 \mathrm{~mol} \%$ at $48 \mathrm{~h}$ and $35.35-44.53$ at $96 \mathrm{~h}$ of enrichment, as well as by their interaction (Table 2, Fig. 2). n-3 / n-6 ratios were ranging between 2.18-3.34 at $24 \mathrm{~h}, 3.18-3.90$ at $48 \mathrm{~h}$ and 3.07-3.10 at $96 \mathrm{~h}$ across the enrichment groups and these ratios in the control group (0 FO) ranged from 2.10 to 2.27 only. Among the analysed FA, statistical differences in the enrichment groups were particularly evident in $n-3$ PUFA $(P<0.05)$, n-6 PUFA, and more importantly in DHA $(P<0.05)$.

The Duncan post hoc test results comparing different enrichment times regardless of dose groups showed significant differences between all fatty acids and groups (Table 2 and Table 3 ) that where manifest as early as the shortest $(24-h)$ enrichment period $(P<0.05)$. A significant increase occurred with $24-h$ enrichment in $\mathrm{n}-3$ PUFA, and the level of $33.94 \mathrm{~mol} \%$ in the control group increased to $34.68 \%, 38.38 \%$ and $44.05 \mathrm{~mol} \%$ in $2.5,5$ and 10 FO-groups, respectively $(\mathrm{P}<0.05)$. Among 
all the analysed FA, the most dramatic change following the 24-h of enrichment was recorded for DHA (Fig. 2). In the worms enriched for $24 \mathrm{~h}$, DHA was measured as 0.92 mol\% in the control group, while the DHA rose to a very striking level of $14.01 \%$ (an increase of 15.23 -fold) with the addition of fish oil at $10 \mathrm{~g}$ per $100 \mathrm{~g}$ of compost $(P<0.05)$. In the lower dose-groups (2.5 and 5 FO, Fig. 2), a sharp increase in DHA was observed only after 48-h of enrichment (15.55-12.51 mol\%). The 96-h enrichment resulted in a further increase in PUFA, n-3 PUFA, EPA and DHA levels. Among the experimental groups, only 2.5 FO-group showed a linear increase in n-3 PUFA from $35.99 \mathrm{~mol} \%$ at $0-\mathrm{h}$ to $44.53 \mathrm{~mol} \%$ at $96-\mathrm{h}$ (Fig. 2). At all of the enrichment levels tested in our study, the worms appeared to reach a DHA saturation level of 14-16 mol\% in 24-48 $\mathrm{h}$ (Fig. 3). In terms of $\mathrm{n}-3$ PUFA, the saturation in worms seemed to be reached at about $45 \mathrm{~mol} \%$ in 5 FO and in 10 FO-groups, whereas this point was not clearly determined in the 2.5 FO-group (Fig. 2).

\section{Discussion}

The first important outcome of this study was the overall fatty acids composition of worms is somewhat comparable to that of fish oil, but with the remarkable exception of DHA, which in worms is at almost trivial levels. This clearly represents an important limiting factor for their potential utilisation in feeding cultured aquatic animals. However, the second important achievement of this study was that of actually showing how DHA content of earthworms can be effectively improved up to nutritionally meaningful levels by a simple enrichment procedure.

In previous studies, it has been reported that earthworms are a suitable feed source for fish in terms of most nutrients, including fatty acids and amino acids (Ignacio et al., 1993; Reinecke et al., 1993; Nandeesha et al., 1988; Istiqomah et al., 2009). Ebadi et al. (2013) compared fish meal with REW in terms of metabolizable energy and fatty acids and found that the energy content of both raw materials was similar but 14:0 and 16:0 fatty acids were higher in the fish meal, while 8:0 to $12: 0$ fatty acids were richer in the earthworms. In general, these researchers have reported that earthworm is a suitable raw material for energy and fatty acids and can be used in feed in terrestrial farm animals, poultry or aquaculture. Hansen \& Czochanska (1975) reported that fatty acids in earthworms are predominantly between 10:0 and 32:0, and that PUFA (especially 18:2n-6 and 18:3n-3) are found at high levels in worms. In another study conducted with REW, 47 $54 \%$ of fatty acids in lipids of worms were reported as $10: 0-24: 0,23 \%$ as MUFA and $13 \%$ as $14: 0-22: 0$ (Kholodova et al., 1978). The clear limiting factor for earthworm potential utilisation is its very limited DHA content, as documented earlier (Kumlu et al., 2018) and confirmed in the present study. This means that, if the worms are to be used directly as feed source for juvenile or broodstock feeding, then the worms will have to be enriched in DHA (Beksari, 2017; Kumlu et al., 2018). Alternatively, if the worms are to be used as major ingredient in feed formulations, then a DHA rich feedstuff (such as FO) must be included in the ration.

Focusing on other nutrients, in a study by Darmawiyanti (2013), it was reported that the overall nutrient content of earthworm (Pheretima sp.) was generally rich, but limited in phospholipid, cholesterol and beta-carotene. It was therefore suggested that when these nutritional deficiencies would have been resolved, they could successfully replace more

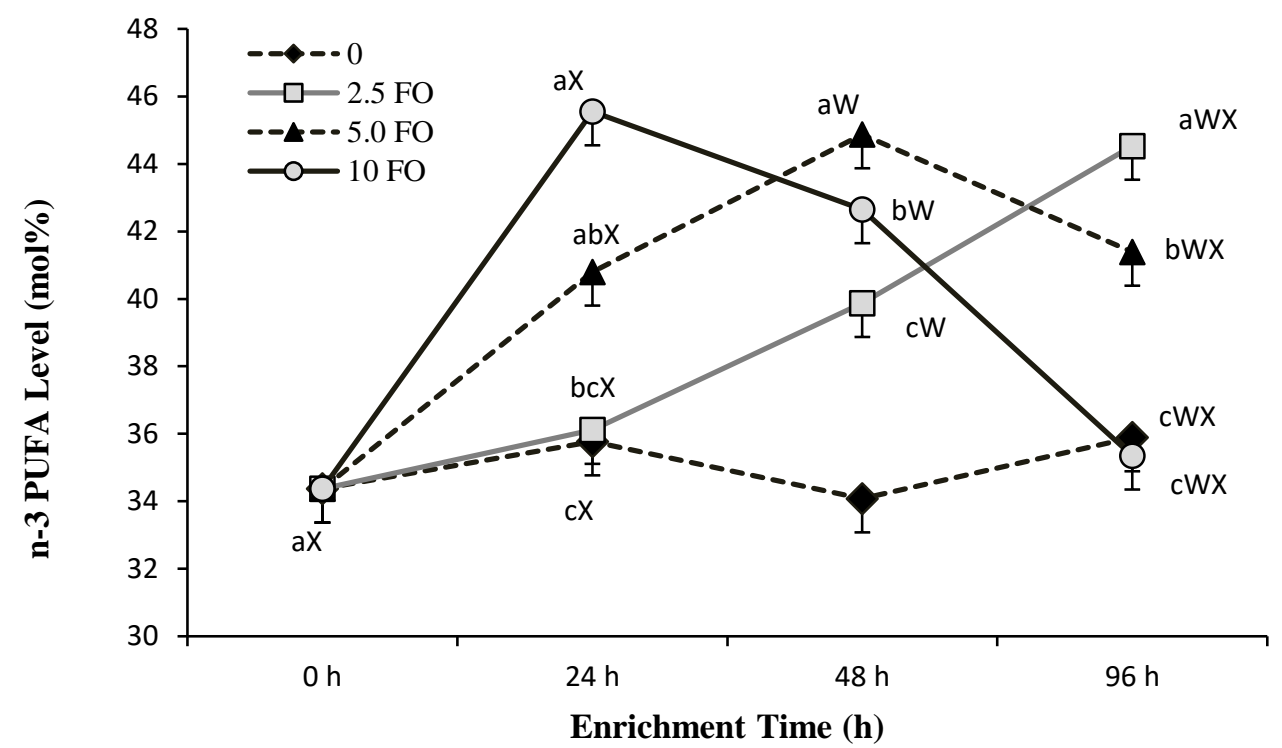

Figure 2. The levels of $n-3$ PUFA in the earthworms Eisenia fetida enriched for up to $96-\mathrm{h}$ with different doses of fish oil $(0,2.5,5.0$ and $10 \% \mathrm{FO})$. Each datapoint represents mean $\pm \mathrm{sd}(\mathrm{mol} \%, \mathrm{n}=3)$. Different lower-case letters (abcd) indicate significant differences $(P<0.05)$ for the same fatty acids/groups among enriching times; different upper-case letters (WXYZ) indicate significant differences $(P<0.05)$ among doses for individual fatty acids/ groups $(P<0.05)$. 


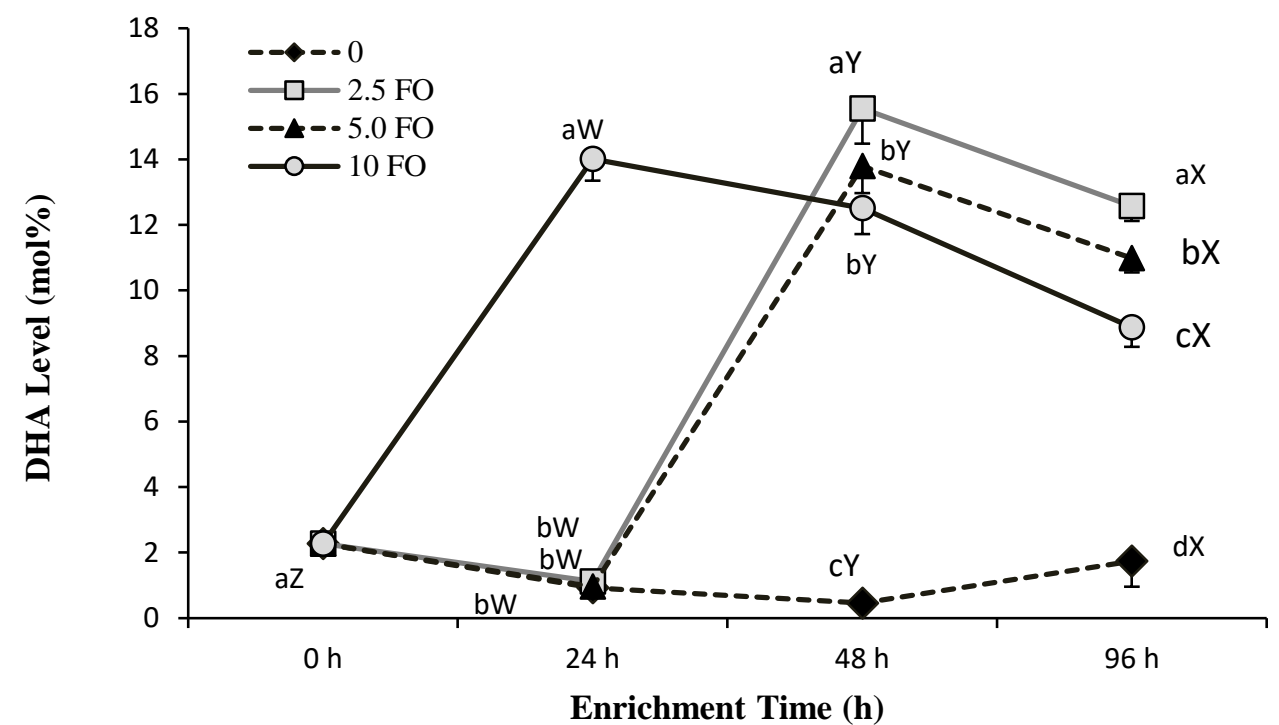

Figure 3. The levels of DHA in the earthworms Eisenia fetida enriched for up to 96 -h with different doses of fish oil $(0,2.5,5.0$ and $10 \% \mathrm{FO})$. Each datapoint represents mean $\pm \mathrm{sd}(\mathrm{mol} \%, \mathrm{n}=3)$. Different lower-case letters (abcd) indicate significant differences $(P<0.05)$ for the same fatty acids/groups among enriching times; different upper-case letters (WXYZ) indicate significant differences $(P<0.05)$ among doses for individual fatty acids/ groups $(P<0.05)$.

expensive marine worms (polychate: Nereis sp.) in the feeding of Penaeus vannamei broodstock. Focusing directly on the most limiting nutrient of earthworm, which appears to be DHA, Kumlu et al., (2018) were the first to demonstrate the potential enrichment of DHA by using a commercial emulsion as a supplement in the compost material for worm culture. In the present follow up study, the potential use of fish oil directly into the culturing compost, as a relatively low cost alternative ingredient replacing a commercial enrichment emulsion, was successfully tested. This study found highly significant effects of fish oil dose, time and interactions (dose $x$ time) among most fatty acids, and in particular DHA of worms. Observing the modification of worm fatty acid composition, as well as the average weight data of the worms measured at $96 \mathrm{~h}$ following the enrichment procedure. Therefore, this trial clearly showed that REW can be enriched to similar LC-PUFA levels as reported by Kumlu et al. (2018) by using fish oil instead of more expensive commercial enrichment products available in the market.

The greatest effects of enrichment with FO on the worms were particularly observed in n-3 PUFA and DHA, whereas more moderate modifications in SFA, MUFA and EPA. Specifically, in the worms enriched for $24 \mathrm{~h}$ with the addition of FO at $10 \mathrm{~g}$ per $100 \mathrm{~g}$ of compost, DHA rose to a very striking level of 14.01 mol\% (an increase of 15-fold) over the control, which possessed the DHA level of only 0.92 mol\% during this period. At all of the enrichment levels tested in our study with FO, the worms appeared to reach a DHA saturation level of around $14-16$ mol\% in 24- $48 \mathrm{~h}$, but the levels fell down at significant rates after $24 \mathrm{~h}$ (10 FO-group) or $48 \mathrm{~h}$ (2.55.0 FO-groups) of enrichments. Similar trends in saturation were also observed in n-3 PUFA levels, except that in 2.5 FO-group displaying a linear trend until the end of enrichment period ( $96 \mathrm{~h}$ ). Enriching REW with a commercial emulsion, Kumlu et al. (2018) found also similar enriched levels of $n-3$ PUFA and DHA contents in the worms in $96 \mathrm{~h}$, but the difference in their results was that the general linear increasing trends of these fatty acids continued until the end of the $96 \mathrm{~h}$-enrichmentperiod indicating that even higher concentrations were possible with further enrichment time. This could likely be due to different nutritional and chemical characteristics of the two enrichment media used in the two studies. Specifically, in the first earthworm enrichment study by Kumlu et al. (2018), the commercial enrichment solution S-Presso (Inve Aquaculture, Dendermonde, Belgium) was used, and this comprised of an aqueous emulsion of a complex lipid matrix, including also trace-elements, microelements, vitamins and proteins. Whereas, in the present study, simple and untreated fish oil, and therefore a triacylglycerols rich oil, was tested. The chemical differences between the two products might therefore being responsible for the differences observed, suggesting that possibly different lipid classes, and/or lipid and other nutrients interactions, might result in different efficiency in the fatty acid uptake capability of earthworms; and this observation clearly warrant further, tailored research experiments towards improving knowledge of lipid metabolism in REW.

Fish oil was previously tested in marine worms, with quite opposite results compared to the present study on earthworms. Jithlang (2007) examined the change in fatty acids content of a marine sand worm ( $P$. nuntia) fed with a feed containing $9 \%$ fish oil twice a week for three months. It was reported that this enrichment approach was not effective in changing fatty 
Table 3. Summary of analysis of variance for fatty acids composition of the earthworms (Eisenia fetida)

\begin{tabular}{|c|c|c|c|c|}
\hline Factor & $d f$ & SeqSS* & F ratio & Prob $>F$ \\
\hline \multicolumn{5}{|l|}{$12: 0$} \\
\hline Doses & 3 & 9.499 & 219.447 & 0.000 \\
\hline Times & 3 & 26.021 & 601.141 & 0.000 \\
\hline Doses X Times & 9 & 33.241 & 255.983 & 0.000 \\
\hline Error & 31 & 0.447 & & \\
\hline \multicolumn{5}{|l|}{$14: 0$} \\
\hline Doses & 3 & 15.917 & 49.935 & 0.000 \\
\hline Times & 3 & 23.927 & 75.065 & 0.000 \\
\hline Doses X Times & 9 & 30.062 & 31.438 & 0.000 \\
\hline Error & 31 & 3.294 & & \\
\hline \multicolumn{5}{|l|}{$16: 0$} \\
\hline Doses & 3 & 11.103 & 532.295 & 0.000 \\
\hline Times & 3 & 7.337 & 351.774 & 0.000 \\
\hline Doses X Times & 9 & 19.957 & 318.930 & 0.000 \\
\hline Error & 31 & 0.216 & & \\
\hline \multicolumn{5}{|l|}{ Other SFA } \\
\hline Doses & 3 & 63.639 & 104.192 & 0.000 \\
\hline Times & 3 & 247.263 & 404.831 & 0.000 \\
\hline Doses X Times & 9 & 40.156 & 21.915 & 0.000 \\
\hline Error & 31 & 6.311 & & \\
\hline \multicolumn{5}{|l|}{ SFA } \\
\hline Doses & 3 & 29.036 & 38.092 & 0.000 \\
\hline Times & 3 & 83.465 & 109.496 & 0.000 \\
\hline Doses X Times & 9 & 206.381 & 90.249 & 0.000 \\
\hline Error & 31 & 7.877 & & \\
\hline \multicolumn{5}{|l|}{$16: 1 n-7$} \\
\hline Doses & 3 & 2.826 & 526.327 & 0.000 \\
\hline Times & 3 & 4.789 & 891.878 & 0.000 \\
\hline Doses X Times & 9 & 4.571 & 283.807 & 0.000 \\
\hline Error & 31 & 0.055 & & \\
\hline \multicolumn{5}{|l|}{$18: 1 n-7$} \\
\hline Doses & 3 & 10.883 & 20.446 & 0.000 \\
\hline Times & 3 & 26.546 & 49.871 & 0.000 \\
\hline Doses X Times & 9 & 8.287 & 5.189 & 0.000 \\
\hline Error & 31 & 5.500 & & \\
\hline \multicolumn{5}{|l|}{$18: 1 n-9$} \\
\hline Doses & 3 & 6.949 & 65.911 & 0.000 \\
\hline Times & 3 & 15.614 & 148.098 & 0.000 \\
\hline Doses X Times & 9 & 20.767 & 65.657 & 0.000 \\
\hline Error & 31 & 1.089 & & \\
\hline \multicolumn{5}{|l|}{ Other MUFA } \\
\hline Doses & 3 & 81.132 & 51.151 & 0.000 \\
\hline Times & 3 & 29.040 & 18.309 & 0.000 \\
\hline Doses X Times & 9 & 91.768 & 19.286 & 0.000 \\
\hline Error & 31 & 16.390 & & \\
\hline \multicolumn{5}{|l|}{ MUFA } \\
\hline Doses & 3 & 67.491 & 100.898 & 0.000 \\
\hline Times & 3 & 41.476 & 62.005 & 0.000 \\
\hline Doses X Times & 9 & 79.307 & 39.521 & 0.000 \\
\hline Error & 31 & 6.912 & & \\
\hline \multicolumn{5}{|l|}{$18: 2 n-6$} \\
\hline Doses & 3 & 41.038 & 166.364 & 0.000 \\
\hline Times & 3 & 93.415 & 378.695 & 0.000 \\
\hline Doses X Times & 9 & 138.754 & 187.499 & 0.000 \\
\hline Error & 31 & 2.549 & & \\
\hline \multicolumn{5}{|l|}{$20: 4 n-6$} \\
\hline Doses & 3 & 0.519 & 15.579 & 0.000 \\
\hline Times & 3 & 0.949 & 28.518 & 0.000 \\
\hline Doses X Times & 9 & 0.823 & 8.241 & 0.000 \\
\hline Error & 31 & 0.344 & & \\
\hline \multicolumn{5}{|l|}{ Other $n-6$ PUFA } \\
\hline Doses & 3 & 2.203 & 22.667 & 0.000 \\
\hline Times & 3 & 10.692 & 109.995 & 0.000 \\
\hline
\end{tabular}




\begin{tabular}{|c|c|c|c|c|}
\hline Doses X Times & 9 & 2.730 & 9.364 & 0.000 \\
\hline Error & 31 & 1.004 & & \\
\hline \multicolumn{5}{|l|}{ n-6 PUFA } \\
\hline Doses & 3 & 76.844 & 10.970 & 0.000 \\
\hline Times & 3 & 67.936 & 9.698 & 0.000 \\
\hline Doses X Times & 9 & 95.275 & 4.534 & 0.001 \\
\hline Error & 31 & 72.385 & & \\
\hline \multicolumn{5}{|l|}{$18: 3 n-3$} \\
\hline Doses & 3 & 32.115 & 74.382 & 0.000 \\
\hline Times & 3 & 150.264 & 348.027 & 0.000 \\
\hline Doses X Times & 9 & 42.034 & 32.452 & 0.000 \\
\hline Error & 31 & 4.462 & & \\
\hline \multicolumn{5}{|l|}{$20: 5 n-3(E P A)$} \\
\hline Doses & 3 & 10.019 & 34.930 & 0.000 \\
\hline Times & 3 & 145.432 & 507.027 & 0.000 \\
\hline Doses X Times & 9 & 14.134 & 16.425 & 0.000 \\
\hline Error & 31 & 2.964 & & \\
\hline \multicolumn{5}{|l|}{$22: 6 n-3(D H A)$} \\
\hline Doses & 3 & 414.225 & 480.939 & 0.000 \\
\hline Times & 3 & 504.059 & 585.242 & 0.000 \\
\hline Doses X Times & 9 & 531.722 & 205.787 & 0.000 \\
\hline Error & 31 & 8.900 & & \\
\hline \multicolumn{5}{|l|}{ Other $n-3$ PUFA } \\
\hline Doses & 3 & 34.004 & 82.252 & 0.000 \\
\hline Times & 3 & 44.049 & 106.548 & 0.000 \\
\hline Doses X Times & 9 & 43.154 & 34.794 & 0.000 \\
\hline Error & 31 & 4.272 & & \\
\hline \multicolumn{5}{|l|}{$n-3$ PUFA } \\
\hline Doses & 3 & 236.596 & 29.093 & 0.000 \\
\hline Times & 3 & 222.696 & 27.384 & 0.000 \\
\hline Doses X Times & 9 & 376.310 & 15.424 & 0.000 \\
\hline Error & 31 & 84.035 & & \\
\hline \multicolumn{5}{|l|}{ PUFA } \\
\hline Doses & 3 & 105.279 & 105.082 & 0.000 \\
\hline Times & 3 & 131.618 & 131.372 & 0.000 \\
\hline Doses X Times & 9 & 391.531 & 130.267 & 0.000 \\
\hline $\begin{array}{l}\text { Error } \\
n-3 / n-6\end{array}$ & 31 & 10.353 & & \\
\hline Doses & 3 & 5.327 & 57.302 & 0.000 \\
\hline Times & 3 & 6.532 & 70.263 & 0.000 \\
\hline Doses X Times & 9 & 5.217 & 18.704 & 0.000 \\
\hline Error & 31 & 0.961 & & \\
\hline \multicolumn{5}{|l|}{$D H A+E P A$} \\
\hline Doses & 3 & 501.292 & 402.830 & 0.000 \\
\hline Times & 3 & 923.007 & 741.713 & 0.000 \\
\hline Doses X Times & 9 & 559.894 & 149.974 & 0.000 \\
\hline Error & 31 & 12.859 & & \\
\hline \multicolumn{5}{|l|}{ DHA/EPA } \\
\hline Doses & 3 & 2.418 & 389.714 & 0.000 \\
\hline Times & 3 & 2.616 & 421.635 & 0.000 \\
\hline Doses X Times & 9 & 3.216 & 172.765 & 0.000 \\
\hline Error & 31 & 0.064 & & \\
\hline
\end{tabular}

*Sequential sum of squares (type III ss)

acid profiles of the marine worms. In a similar study, when Klinchoedchue et al. (2011) fed the above worm species on a diet containing $16.7 \% \mathrm{FO}$, they found an increase in ARA (20:4n-6) and EPA, but not in DHA. These researchers concluded that the marine sand worms should be fed for at least 26 days on the enriched feed to change some of their fatty acids content. Fairchild et al. (2017) reported that the marine worm Enchytraeus albidus contained lower n-3 PUFA, similar EPA and LC-
PUFA compared to Artemia, rotifer and copepods and that its DHA content was very low $(0-0.5 \%)$. These researchers found that feeding these worms on various feeds were effective on tissue fatty acids composition, but causing only small increases in DHA contents. Hence, the results of the present study on red earthworms and the available literature comparisons on marine worms clearly indicate that the DHA metabolism, and its ability to be accumulated into worm's body, is remarkably 
different between worms living in the two different environments. Therefore, it can also be concluded that, in terms of fatty acids profiles, marine worms -with or without enrichment- do not have any superior nutritive properties, and actually could be less nutritionally beneficial, when compared to DHA enriched REW. Nevertheless, there is ample scientific evidence of the potential of marine worms as feed for aquatic animals. Bray \& Lawrence (1992), and Oddsen (2014) found that the use of marine worms (Nereis virens) in the feeding of whiteleg shrimp broodstock resulted in higher nauplii production per female and that the larval growth and survival obtained from these females increased due to LC-PUFA, prostaglandins and bromophenols present in the contents of marine worms (Lytle et al., 1990). Wouters et al. (1999) recommended an n-3 / n-6 PUFA ratio of 2-3, while Palacios et al. (1999) showed that triacylglycerols meet the basic energy needs of shrimp embryos and nauplii and are effective on reproduction, egg and postlarval quality. Studies conducted with different shrimp species suggest that the content of LCPUFA in feeds should be between 0.5 and 1\% (Kanazawa et al., 1979a; Xu et al., 1994). Thus, based on the above reports on marine worms and the results of the present enrichment study in earthworm, it can be concluded that REW has great potential for utilisation as aquaculture feed, albeit requiring an enrichment process. Therefore, future research studies should focus on testing the performance of cultured aquatic animals fed DHA enriched earthworms, and also possibly investigating if they contain other beneficial micronutrients that might stimulate reproduction, as reported for marine worms (Lytle et al., 1990). Importantly, future studies on earthworms should also consider other factors, such as the presence or absence of risks associated with disease transmission to cultured stock, as observed for other live food used in aquaculture (Velu \& Munuswamy, 2004; Kostecka \& Paczka, 2006; Tziouveli et al., 2012).

The red earthworms have been showed to be readily accepted and consumed by the Pacific white shrimp $P$. vannamei and the redclaw crayfish Cherax quadricarinatus (Beksari, 2017). This study also showed that REW can survive up to at least $48 \mathrm{~h}$ in freshwater at $25^{\circ} \mathrm{C}$, and can resist $39 \%$ salinity for about $3 \mathrm{~min}$, providing to be an effective bait/live feed. Now that the fatty acid profile of the earthworms has been shown to be efficiently manipulated by using either commercial enrichment media (Kumlu et al., 2018), or simply fish oil (the present study), the resulting effects on broodstock performance of fish / shrimp should be fully tested in further studies. Additionally, given the present study showed that earthworms can efficiently utilise and deposit DHA from triacylglycerols, it is likely that even cheaper source of DHA can be effectively utilised in their compost material, such as fish and seafood processing by-products. This will allow earthworm cultivation to potentially contribute to the current global effort in improving resource sustainability, nutrient recycling and minimisation of food wastes.

In conclusion, this study showed that REW can be efficiently enriched to contain high levels of $n-3$ LCPUFA, and especially DHA, using only fish oil in their compost material. The results of the study also indicate that 2.5-5 g of fish oil inclusion per $100 \mathrm{~g}$ of compost is suitable for enrichment of the worm REW for 48-96 $h$, but if more rapid enrichment is desired (i.e. $24 \mathrm{~h}$ ), then up to $10 \mathrm{~g}$ FO inclusion per $100 \mathrm{~g}$ of compost may be recommended. The present study therefore contributes to gain knowledge necessary for establishing REW as a high potential candidate as aquaculture feed, in the form of live prey, or even in other forms such as frozen or freeze-dried, particularly for early larval/post-larval stages or freshwater/marine broodstock.

\section{Ethical Statement}

All animal handling procedures used in the present study was fully compliant with the Turkish guidelines for animal care (No. 28141), as set by the Ministry of Food, Agriculture and Livestock.

\section{Funding Information}

This study was supported by the Scientific Research Fund of Cukurova University (Grant No: FDK2016-6272).

\section{Author Contribution}

M. Kumlu: Experiment design, Data curation, Formal Analysis, Investigation, Methodology, Writing original draft; Writing - review \& editing, A. Beksarı: Experiment design, Data curation, Investigation,Writing - review \& editing; H.A. Yılmaz: Data curation, Formal Analysis,Investigation, Writing- Original draft, Writing - review \& editing; M. Sariipek: ,Investigation; , Writing - review \& editing; E. Kınay: Data curation; Investigation; G. Turchini: Data curation; Writing original draft; Writing - review \& editing. O. T. Eroldoğan: Supervision, Conceptualization, Data curation, Writing - original draft; Writing - review \& editing. All authors approved the submission and publication of this manuscript.

\section{Conflict of Interest}

There is no conflict of interest regarding the submission and publication of this manuscript, financial or non-financial.

\section{References}

Arts, M. T., Ackman, R. G., \& Holub, B. J. (2001). " Essential fatty acids" in aquatic ecosystems: a crucial link between diet and human health and evolution. Canadian Journal of 
Fisheries and Aquatic Sciences, 58(1), 122-137. 7. https://doi.org/10.1139/f00-224

Bahadori, Z., Esmaylzadeh, L., \& Torshizi, M. A. K. (2015). The effect of earthworm (Eisenia fetida) and vermihumus meal in diet on broilers chicken efficiency and carcass components. In Biological Forum (Vol. 7, No. 1, p. 998). Research Trend.

Beksari, A. (2017). Enrichment of HUFA contents of the earthworm Eisenia fetida and it's use as feed for the shrimp Penaeus vannamei. PhD Thesis. Cukurova University, Adana-Turkey, $178 \mathrm{pp}$.

Bray, W.A., \& Lawrence, A.L. (1992). Reproduction of Penaeus species in captivity. In: Fast, A.W., Lester, J.L. (Eds.), Marine Shrimp Culture: Principles and Practices. Elsevier, Amsterdam pp. 93-170.

Castro, L. F. C., Tocher, D. R., \& Monroig, O. (2016). Long-chain polyunsaturated fatty acid biosynthesis in chordates: Insights into the evolution of Fads and Elovl gene repertoire. Progress in lipid research, 62, 25-40. https://doi.org/10.1016/j.plipres.2016.01.001

Chauhan, A., Kumar, S., Singh, A. P., \& Gupta, M. (2010). Vermicomposting of vegetable wastes with cowdung using three earthworm species Eisenia foetida, Eudrilus eugeniae and Perionyx excavatus. Nature and science, $8(1), 34-42$.

Chiu, S. T., Wong, S. L., Shiu, Y. L., Chiu, C. H., Guei, W. C., \& Liu, C. H. (2016). Using a fermented mixture of soybean meal and earthworm meal to replace fish meal in the diet of white shrimp, Penaeus vannamei (Boone). Aquaculture Research, 47(11), 3489-3500.

https://doi.org/10.1111/are.12799

Czesny, S., \& Dabrowski, K. (1998). The effect of egg fatty acid concentrations on embryo viability in wild and domesticated walleye (Stizostedion vitreum). Aquatic Living Resources, 11(6), 371-378.

https://doi.org/10.1016/S0990-7440(99)80002-3

Darmawiyanti, V. (2013). The evaluation of earthworm (Pheretima sp) enrichment on the chemical composition and ovarian development of female Pacific white shrimp (Litopenaeus vannamei) broodstock. Master Thesis, Sekolah Pasca Sarjana Institut Pertanian Bogor, Indonesia, pp. 40.

Ebadi, Z., Mirhadi, S.A., \& Yaghobfar, A. (2013). Comparison of the metabolizable energy and fatty acidp rofile of earthworm meal (Eisenia fetida) with fish meal (domestic and imported). Iranian Animal Science Reseaches Journal 9, 24-29.

Fairchild, E. A., Bergman, A. M., \& Trushenski, J. T. (2017). Production and nutritional composition of white worms Enchytraeus albidus fed different low-cost feeds. Aquaculture, 481, 16-24. https://doi.org/10.1016/j.aquaculture.2017.08.019

Folch, J., Lees, M., \& Stanley, G. S. (1957). A simple method for the isolation and purification of total lipides from animal tissues. Journal of biological chemistry, 226(1), 497-509.

Hansen, R.P., \& Czochanska, Z. (1975). The fatty acid composition of the lipids of earthworms. J. Sci. Food Agric. 26, 961-971. https://doi.org/10.1002/jsfa.2740260713

Honnens, H., Assheuer, T., \& Ehlers, R. U. (2014). Enrichment of the nematode Panagrolaimus sp., a potential live food for marine aquaculture, with essential n-3 fatty acids. Aquaculture international, 22(2), 399-409. https://doi.org/10.1007/s10499-013-9648-3
Ignacio, A., Carlos, A., Luois, A., \& Hebel, P. (1993). Nutritional and toxicological evaluation of earthworm (Eisenia foetida) meal as protein source for animal feed. Animal Feed Science and Technology 42, 165-172. https://doi.org/10.1016/0377-8401(93)90031-E

Istiqomah, L., Sofyan, A., Damayanti, E., \& Julendra, H. (2009). Amino acid profil of earthworm and earthworm meal (Lumbricus rubellus) for animal of dstuff. Journal of the Indonesian Tropical Animal Agriculture, 34(4), 253-257.

Jithlang, I. (2007). Lipid and Vitamin E Enrichment in Sand Worm (Perinereis nuntia, Savigny). Coastal Fisheries Research and Development Bureau, Department of Fisheries Ministry of Agriculture and Cooperatives, Thailand.

http://www.lib.ku.ac.th /kuconf/kc4504039.pdf

Jobling, M. (2016). Fish nutrition research: past, present and future. Aquaculture international, 24(3), 767-786. https://doi.org/10.1007/s10499-014-9875-2

Kanazawa, A., Teshima, S., \& Ono, K. (1979a). Relationship between essential fatty acid requirements of aquatic animals and the capacity for bioconversion of linolenic acid to highly unsaturated fatty acids. Comparative Biochemistry and physiology. B, Comparative Biochemistry, 63(3), 295-298. https://doi.org/10.1016/0305-0491(79)90251-7

Kanazawa, A., Teshima, S., Ono. K., \& Chalayodeja, K. (1979b). Biosynthesis of fatty acids from acetate in the prawn, Penaeus monodon and Penaeus merguiensis. Mem. Fac. Fish. Kagoshima Univ. 28, 21-26.

Kholodova, I., Mironova, V.N., Povkhan, M.F., Golodniĭ, N.M., Berdys-Hev, A.G., BulevskiĬ, N.V., \& Mel'nik, I.A. (1978.) Lipid and fatty acid composition of Eisenia foetida. Ukr. Biokhim. Zh. 63(3), 76-81.

Klinchoedchue, P., Jithlang, I., \& Samranrat, N. (2011). Optimum Duration for Tuna Oil and Vitamin E Enrichment in Sand Worm Feed (Perinereis nuntia, Savigny 1818). Technical Paper No. 23/2011, Coastal Fisheries Research and Development Bureau, Department of Fisheries Ministry of Agriculture and Cooperatives, Thailand.

Kostecka, J., \& Pączka, G. (2006). Possible use of earthworm Eisenia fetida (Sav.) biomass for breeding aquarium fish. European Journal of Soil Biology 42, 231-233. https://doi.org/10.1016/j.ejsobi.2006.07.029

Kumlu, M., Beksari, A., Eroldoğan, O. T., Yılmaz, H. A., Sarripek, M., Kınay, E., \& Turchini, G. M. (2018). DHA enrichment of the red earthworm Eisenia fetida for improving its potential as dietary source for aquaculture. Aquaculture, 496, 10-18. https://doi.org/10.1016/j.aquaculture.2018.07.005

Kumlu, M., Fletcher, D. J., \& Fisher, C. M. (1998). Larval pigmentation, survival and growth of Penaeus indicus fed the nematode Panagrellus redivivus enriched with astaxanthin and various lipids. Aquaculture Nutrition, 4(3), 193-200.

https://doi.org/10.1046/j.1365-2095.1998.00071.x

Langer, S., Bakhtiyar, Y., \& Lakhnotra, R. (2011). Replacement of fishmeal with locally available ingredients in diet composition of Macrobrachium dayanum. African J. Agricultural Research 6(5), 1080-1084. https://doi.org/10.5897/AJAR.9000588

Latsamy, P., \& Preston, T. R. (2007). Fly larvae, earthworms and duckweed as feeds for frogs in an integrated farming system (Doctoral dissertation, MSc Thesis, MEKARN-SLU 
http://www. mekarn. org/MSC2005-07/thesis07/lasts2. htm).

Leger, P., \& Sorgeloos, P. (1992). Optimized feeding regimes in shrimp hatcheries. Developments in aquaculture and fisheries science, 23, 225-24

Liu, S. L., Liu, Y., Chen, M. Y., Zhou, Y., Zhang, T., \& Yang, H. S. (2008). Effects of fresh diet Eisenia foetida on growth ad biochemical components of Fenneropenaeus chinensis juveniles. J. Fish. Sci. China, 1, 145-153.

Lytle, J.S., Lytle, T.F., \& Ogle, J. (1990). Polyunsaturated fatty acid profiles as a comparative tool in assessing maturation diets of Penaeus setiferus. Aquaculture 89, 287-299. https://doi.org/10.1016/0044-8486(90)901338

Mason, W.T., Rottmann, R.W., \& Dequine, J.F. (1992). Culture of earthworms for bait or fish food. University of Florida Extension CIR1053, pp. 1-4.

Medale, F., \& Kaushik, S. (2009). Protein sources in feed for farmed fish. Cahiers Agricultures 18(2), 103111. https://doi.org/10.1684/agr.2009.0279

Metcalfe, L., \& Schmitz, A. (1961). The lipid preparation of fatty acid esters for gas chromatographic analysis. Anal. Chem. 33, 363-364.

Mutti, D. W., Ballester, E. L., Martino, R. C., Wasielesky, W., \& Cavalli, R. O. (2017). Feeding n-3 HUFA enriched Artemia to the larvae of the pink shrimp Farfantepenaeus paulensis increases stress tolerance and subsequent growth. Latin american journal of aquatic research, 45(1), 18-24. http://dx.doi.org/10.3856/vol45-issue1fulltext-2

Musyoka, S. N., Liti, D. M., Ogello, E., \& Waidbacher, H. (2019). Utilization of the earthworm, Eisenia fetida (Savigny, 1826) as an alternative protein source in fish feeds processing: A review. Aquaculture Research, 50(9), 2301-2315. https://doi.org/10.1111/are.14091

Nandeesha, M.C., Srikanth, G.K., Basavaraja, N., Keshavanath, P., Varghese, T.J., Bano, K., Ray, A.K., \& Kale, R.D. (1988). Influence of earthworm meal on the growth and flesh quality of common carp. Biological Wastes 26, 189-198. https://doi.org/10.1016/0269-7483(88)90165-6

National Research Council (NRC). (2011). Nutrient requirements of fish and shrimp. National academies press.

Oddsen, O. (2013). Polychaetes as valuable fish feed ingredient.https://www.allaboutfeed.net/FeedAdditives/Articles/2014/11/Polychaetes-as-valuablefish-feed-ingredient-1556050W/

Palacios, E., Perez-Rostro, C.I., Ramirez, J.L., Ibarra, A.M., \& Racotta, I.S. (1999). Reproductive exhaustion in shrimp Penaeus vannamei reflected in larval biochemical composition, survival and growth. Aquaculture 171, 309321. https://doi.org/10.1016/S0044-8486(98)00393-7

Paoletti, M. G., Buscardo, E., VanderJagt, D. J., Pastuszyn, A., Pizzoferrato, L., Huang, Y. S., \& Glew, R. H. (2003). Nutrient content of earthworms consumed by Ye'Kuana Amerindians of the Alto Orinoco of Venezuela. Proceedings of the Royal Society of London. Series B: Biological Sciences, 270(1512), 249-257. https://doi.org/10.1098/rspb.2002.2141

Parrillo, L., Coccia, M., Volpe, M.G., Siano, F., Pagliarulo, C., Scioscia, E., Varricchio, E., Safari, O., Eroldogan, T., \& Paolucci, M. (2017). Olive mill wastewater-enriched diet positively affects growth, oxidative and immune status and intestinal microbiota in the crayfish, Astacus leptodactylus. Aquaculture 473, 161-168. https://doi.org/10.1016/j.aquaculture.2017.02.013

Reinecke, A.J., Hayes, J.P., \& Cilliers, S.C. (1991). Protein quality of three different species of earthworms. South African Journal of Animal Science 21(2), 99-103.

Rezaeipour, V., Nejad, O. A., \& Miri, H. Y. (2014). Growth performance, blood metabolites and jejunum morphology of broiler chickens fed diets containing earthworm (Eisenia foetida) meal as a source of protein International Journal of Advanced Biological and Biomedical Research, 2(8), 2483-2494.

Sargent, J. R., Bell, J. G., Bell, M. V., Henderson, R. J., \& Tocher, D. R. (1995). Requirement criteria for essential fatty acids. Journal of applied Ichthyology, 11(3/4), 183198.https://doi.org./10.1111/j.14390426.1995.tb00018.x

Sorgeloos, P., Dhert, P., Candreva, P. 2001. Use of the brine shrimp, Artemia spp., in marine fish larviculture. Aquaculture200,147-159. https://doi.org/10.1016/S0044-8486(01)00698-6

Stafford, E. A., \& Tacon, A. G. (1985). The nutritional evaluation of dried earthworm meal (Eisenia foetida, Savigny, 1826) included at low levels in production diets for rainbow trout, Salmo gairdneri Richardson. Aquaculture Research, 16(3), 213-222. https://doi.org/10.1111/j.1365-2109.1985.tb00310.x

Suprayudi, M. A., Takeuchi, T., \& Hamasaki, K. (2004). Effects of Artemia enriched with eicosapentaenoic and docosahexaenoic acid on survival and occurrence of molting failure in megalop larvae of the mud crab Scylla serrata. Fisheries science, 70(4), 650-658. https://doi.org/10.1111/j.1444-2906.2004.00853.x

Tacon, A.G.J., Metian, M. 2009. Fishing for aquaculture: Nonfood use of small pelagic forage fish-a global perspective. Revies in Fisheries Science 17, 305-317. https://doi.org/10.1080/10641260802677074

Tacon, A. G. J., Stafford, E. A., \& Edwards, C. A. (1983). A preliminary investigation of the nutritive value of three terrestrial lumbricid worms for rainbow trout. Aquaculture, 35, 187-199. https://doi.org/10.1016/0044-8486(83)90090-X

Takeuchi, T., Nakamoto, Y., Hamasaki, K., Sekiya, S., \& Watanabe, T. (1999). Requirement of $\mathrm{N}-3$ highly unsaturated fatty acids for larval swimming crab Portunus trituberculatus. Nippon Suisan Gakkaishi, 65(5), 797-803.

Turchini, G.M. (2013). Fish Oils, Misconceptions and the Environment. American Journal of Public Health 103(11), e4-e5. https://dpi.org./10.2105/AJPH.2013.301510

Turchini, G.M., Torstensen, B.E., Ng, W.K. 2009. Fish oil replacement in finfish nutrition. Reviews in Aquaculture 1, 10-57. https://doi.org/10.1111/j.1753-5131.2008.01001.x

Tziouveli, V., Hall, M., \& Smith, G.G. (2012). Evaluation of lipidenriched Artemia on the reproductive performance of the white-striped cleaner shrimp, Lysmata amboinensi. Aquacult. Int. 20, 201-211. https://doi.org/10.1007/s10499-011-9496-y

Velu, C. S., \& Munuswamy, N. (2004). Improving the fatty acid profile of fairy shrimp, Streptocephalus dichotomus, using a lipid emulsion rich in highly unsaturated fatty acids. Journal of agricultural and food chemistry, 52(23), 7033-7038. https://doi.org/10.1021/jf0490605

Watanabe, T. (1993). Importance of docosahexaenoic acid in marine larval fish. Journal of the World Aquaculture 
Society, 24(2), 152-161. https://doi.org/10.1111/j.17497345.1993.tb00004.x

Vodounnou, D. S., Juste, V., Kpogue, D. N. S., Apollinaire, M. G., \& Didier, F. E. (2016). Culture of earthworm (Eisenia fetida), production, nutritive value and utilization of its meal in diet for Parachanna obscura fingerlings reared in captivity. International Journal of Fisheries and Aquatic Studies, 4(5), 01-05.

Wouters, R., Gomez, L., Lavens, P., \& Calderon, J. (1999). Feeding enriched Artemia biomassa to Penaeus vannamei broodstock: its effect on reproductive performance and larval quality. J. Shellfish Res. 18, 651656. http://hdl.handle.net/1854/LU-170411

Xu, X.L., Ji, W.J., Castell, J.D., \& O'dor, R.K. (1994). Effect of dietary lipid sources on fecundity, egg hatchability and egg fatty acid composition of Chinese prawn (Penaeus chinensis) broodstock. Mar. Fish. Res. 13, 13-19. https://doi.org/10.1016/0044-8486(94)90300-X

Yaqub, H.B. 1997. Earthworm and maggot meals as a potential fishmeal replacement.

http://www.oceandocs.org/bitstream 